\title{
Article \\ Differential Phase Shift Quantum Secret Sharing Using a Twin Field with Asymmetric Source Intensities
}

\author{
Zhao-Ying Jia, Jie Gu, Bing-Hong Li, Hua-Lei Yin * ${ }^{\circ}$ and Zeng-Bing Chen * \\ National Laboratory of Solid State Microstructures, School of Physics and Collaborative Innovation Center of \\ Advanced Microstructures, Nanjing University, Nanjing 210093, China; MG20220091@smail.nju.edu.cn (Z.-Y.J.); \\ MG20220083@smail.nju.edu.cn (J.G.); 171830542@smail.nju.edu.cn (B.-H.L.) \\ * Correspondence: hlyin@nju.edu.cn (H.-L.Y.); zbchen@nju.edu.cn (Z.-B.C.)
}

Citation: Jia, Z.-Y.; Gu, J.; Li, B.-H.; Yin, H.-L.; Chen, Z.-B. Differential Phase Shift Quantum Secret Sharing Using a Twin Field with Asymmetric Source Intensities. Entropy 2021, 23, 716. https://doi.org/10.3390/ e23060716

Academic Editors: Remigiusz Augusiak and Marcin Pawłowski

Received: 4 May 2021

Accepted: 1 June 2021

Published: 4 June 2021

Publisher's Note: MDPI stays neutral with regard to jurisdictional claims in published maps and institutional affiliations.

Copyright: (c) 2021 by the authors. Licensee MDPI, Basel, Switzerland. This article is an open access article distributed under the terms and conditions of the Creative Commons Attribution (CC BY) license (https:// creativecommons.org/licenses/by/ $4.0 /)$.

\begin{abstract}
As an essential application of quantum mechanics in classical cryptography, quantum secret sharing has become an indispensable component of quantum internet. Recently, a differential phase shift quantum secret sharing protocol using a twin field has been proposed to break the linear rate-distance boundary. However, this original protocol has a poor performance over channels with asymmetric transmittances. To make it more practical, we present a differential phase shift quantum secret sharing protocol with asymmetric source intensities and give the security proof of our protocol against individual attacks. Taking finite-key effects into account, our asymmetric protocol can theoretically obtain the key rate two orders of magnitude higher than that of the original protocol when the difference in length between Alice's channel and Bob's is fixed at $14 \mathrm{~km}$. Moreover, our protocol can provide a high key rate even when the difference is quite large and has great robustness against finite-key effects. Therefore, our work is meaningful for the real-life applications of quantum secret sharing.
\end{abstract}

Keywords: quantum cryptography; quantum secret sharing; differential phase shift

\section{Introduction}

Secret sharing is a cryptographic protocol in which a dealer splits a secret into several parts and distributes them among various players. The secret can be recovered only when a sufficient number of players (authorized subsets) cooperate to share their parts of the secret. The classical secret sharing scheme was first introduced independently by Shamir [1] and Blakley [2] in 1979, followed by plenty of variations [3]. However, all existing classical secret sharing schemes are not perfectly secure from eavesdropping attacks [4].

As the combination of classical secret sharing and quantum mechanics, quantum secret sharing (QSS) is more secure due to the excellent properties of quantum theory and has become one of the most attractive research topics in the quantum cryptography. In 1999, Hillery et al. [5] firstly proposed a protocol of QSS using a three-photon GreenbergerHorne-Zeilinger (GHZ) state. Afterwards, this protocol was generalized into an arbitrary number of parties based on multi-particle entanglement states [6], and later to multiparticle $d$-dimensional entanglement states [7]. From then on, much theoretical [8-15] and experimental [16-19] attention has focused on QSS using multi-particle entangled states. However, it is a tremendous challenge to prepare a multiparty entanglement state with high fidelity and efficiency, which makes particle entanglement-based QSS unscalable. To circumvent the problems, differential phase shift QSS scheme using coherent light [20], similar to those used in quantum key distribution (QKD) [21-26], has been proposed and implemented.

Nevertheless, the linear rate-distance limitation constricts the key rate and transmission distance of QSS $[27,28]$. Recently, to exceed the linear bound and further enhance the practical performance of QSS, a differential phase shift quantum secret sharing (DPSQSS) protocol [29] using a twin field (TF) [30] has been proposed. Unfortunately, this protocol 
suffers from low key rate and short transmission distance over channels with different transmittances, which constrains its application in a practical network setting.

Here, we propose an asymmetric differential phase shift quantum secret sharing protocol using twin field [30-44] ideas and give the security proof of this protocol against individual attacks. The key point of our method is that Alice and Bob can adjust their source intensities independently to effectively compensate for channel asymmetry. The numerical results show that our protocol is robust against finite-key effects [45-48] and can theoretically provide a two orders of magnitude higher key rate than the original protocol with the length difference between Alice's channel and Bob's fixed at $14 \mathrm{~km}$. Furthermore, our protocol still obtains a high key rate when the difference in length is fixed at 50,100 km, whereas no keys are obtained with the same difference in the original protocol. Therefore, our work represents a further step along the progress of practical QSS.

\section{TF-DPSQSS Protocol with Asymmetric Source Intensities}

The schematic diagram of our asymmetric protocol is shown in Figure 1, where Alice and Bob have partial keys for deciphering, and Charlie has a full key for ciphering. The two senders, Alice and Bob, independently prepare two trains of weak coherent pulses whose intensities are different and phases are randomly modulated to be 0 or $\pi$. The coherent pulses are sent through the quantum channels and received by the trusted third party, Charlie, who measures them using an unbalanced interferometer. The details of the protocol are shown as follows.

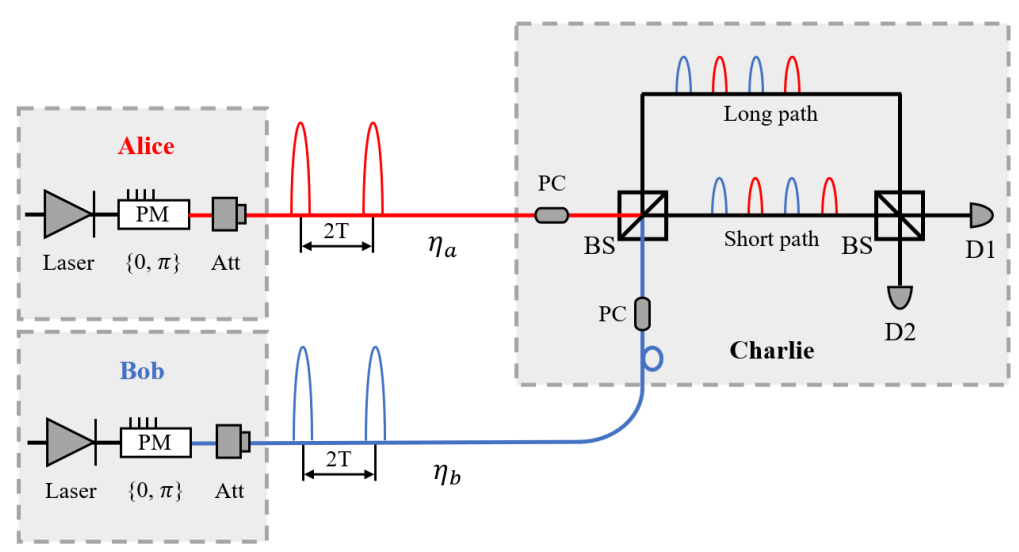

Figure 1. Configuration of our quantum secret sharing protocol.Weak coherent pulse sources (Laser); phase modulator (PM); signal attenuator (Att); transmittance of channels between Alice (Bob) and Charlie $\left(\eta_{a}, \eta_{b}\right)$; polarization control (PC); beam splitter (BS); single photon detector (D1, D2). In Charlie's measurement area, two pulse trains are first polarization-modulated by polarization controls to correct their polarization for interference. Then, Charlie divides each incoming pulse into two paths and recombines them by a 50:50 beam splitter, where the path-length difference is set equal to the time T. Detectors are placed at the two outputs of the recombining beam splitter. At the detectors, the partial wave functions of two senders' pulses that are in the same time slots interfere with each other.

Preparation: Alice (Bob) prepares a weak coherent pulse train and phase-modulates each pulse randomly by 0 or $\pi$. The coherent states can be denoted as $\left|\psi_{a}\right\rangle=\underset{n=1}{\otimes}\left|\sqrt{\mu_{a}} e^{i \phi_{n}^{a}}\right\rangle$ $\left(\left|\psi_{b}\right\rangle=\stackrel{\otimes}{n=1}_{n}^{N}\left|\sqrt{\mu_{b}} e^{i \phi_{n}^{b}}\right\rangle\right)$. Then, she (he) sends out the coherent state pulse whose period is $2 \mathrm{~T}$ to Charlie with an average photon number less than one per pulse. Alice (Bob) records her (his) logic bits of each time slot as " 0 " (" 1 ") when her (his) modulated phase is $0(\pi)$. We denote the sequence of detection events time slots as $n \in\{1,2,3, \ldots, 2 N\}$, where $N$ is the total number of pulses sent by Alice (Bob). The phase shift $\phi_{n}^{a}\left(\phi_{n}^{b}\right) \in\{0, \pi\}$ is the 
phase induced by the phase modulator on pulse $n$ ignoring the global phase, and intensities $\mu_{a}, \mu_{b}$ are corresponding to Alice and Bob, respectively.

Measurement: As illustrated in Figure 1, while measuring the signal, Charlie records the photon detection time and which detector clicks. When the detection event time slots are corresponding to the time $2 k T$, detector 1 will click for 0 phase difference between the two senders' pulses and detector 2 will click for $\pi$ phase difference. When the detection event time slots are corresponding to the time $(2 k+1) T$, detector 1 will click for $\pi$ phase difference and detector 2 will click for 0 phase difference. Note that if both detectors click, Charlie randomly chooses one detector click to record. Here, a photon is detected occasionally and randomly because the received signal power is smaller than one photon per pulse.

Using the above setup, Charlie creates their key shown in Table 1. " 0 " means that the modulated phases in one time slot imposed by Alice and Bob are $\{0,0\}$ or $\{\pi, \pi\}$, and " 1 " means that the modulated phases in one time slot imposed by Alice and Bob are $\{0, \pi\}$ or $\{\pi, 0\}$. Thus, Charlie's bits are exclusive OR of Alice's and Bob's bits. That is, Alice and Bob know Charlie's key bits only when they cooperate, and the QSS operation is accomplished.

Table 1. Logic bits held by Charlie corresponding to different detection event time slots when detector 1 or detector 2 clicks.

\begin{tabular}{lcc}
\hline & Detector 1 & Detector 2 \\
\hline $2 k T$ & "0" & " $1 "$ \\
$(2 k+1) T$ & "1" & " 0 " \\
\hline
\end{tabular}

Parameter estimation: Charlie randomly chooses recorded detection times and Alice and Bob alternatively disclose her or their test bit first in the chosen time slots through a public channel. Then, Charlie will get the quantum bit error rate (QBER) and make a decision whether they discard all their bits and restart the whole QSS at Step 1 (preparation).

Postprocessing: After calculating the QBER, Alice, Bob, and Charlie will conduct classical error correction and privacy amplification to distill the final full key and partial keys.

\section{Proof of Security}

In this section, we will discuss the security of our protocol against eavesdropping. Because of the equivalence [29] between our asymmetric protocol and differential phase shift QSS, we can apply the conclusion in differential phase shift quantum key distribution [25] to the analysis of both an external eavesdropper and an internal eavesdropper in our protocol.

\subsection{External Eavesdropping}

Firstly, Eve cannot obtain full key information by beam-splitting attacks and interceptresend attacks, which will result in bit errors in the secret key [22]. As for a general individual attack, based on the assumption that Eve will conduct the same attack in differential phase shift QSS [20], we can derive that information leakage to Eve is given by a fraction $\mu_{a}\left(1-\eta_{a}\right)+\mu_{b}\left(1-\eta_{b}\right)$ of the sifted key [25].

\subsection{Internal Eavesdropping}

QSS protocols have to prohibit Alice or Bob from knowing Charlie's key by herself or himself. Firstly, we assume that Bob is the malicious one. In this case, we have equivalence configuration [29] shown in Figure 2a. Bob wants to know Charlie's key by himself, and also needs to know Alice's modulation phase to pass the test-bit checking among Alice, Bob, and Charlie after the creation of the raw key. A configuration for Bob to do so is shown in Figure 2b. He conducts general individual attacks as carried out by Eve in differential phase shift quantum key distribution [25], where the fraction Bob obtains about Charlie's bits is $2 \mu_{a}$. Similarly, when Alice is the malicious one, the probability that Alice knows 
Bob's differential phase corresponding to Charlie's bit is $2 \mu_{b}$. From the above discussion, we denote $\mu_{\max }=\max \left\{\mu_{a}, \mu_{b}\right\}$ as the maximum intensity, then $2 \mu_{\max }$ is the maximum ratio of information that a malicious one can obtain from the internal eavesdropping.

(a)

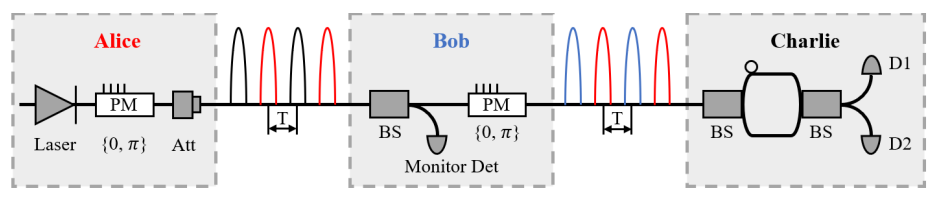

(b)

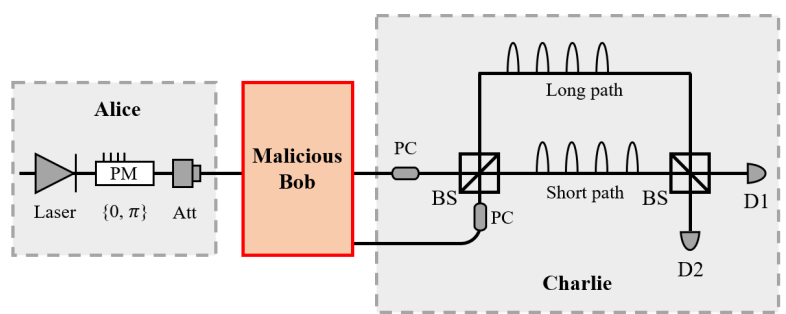

Figure 2. Internal eavesdropping of malicious Bob. (a) With some special rules discussed in [29], we present the configuration of equivalence between differential phase shift QSS and our protocol. (b) We present the configuration of a general individual attack by malicious Bob.

In conclusion, we find that the probability information leakage to Eve and malicious Bob (Alice) is $\mu_{a}\left(1-\eta_{a}\right)+\mu_{b}\left(1-\eta_{b}\right)$ and $2 \mu_{a}\left(2 \mu_{b}\right)$. We discover that information leakage to external Eve is slightly lower than that to malicious Bob (Alice). For simplicity, we can just consider information leakage in our protocol to be $2 \mu_{\max }$.

\section{Numerical Simulation}

\subsection{Mathematical Calculation with Asymmetric Channels}

Based on the asymmetric protocol description, Charlie generates their classical bits according to the phase differences between Alice and Bob in the same time slot. To obtain the secure key rate, we apply the same denotations in previous sections that Alice and Bob send pulses with intensities $\mu_{a}, \mu_{b}$, and the distance between Alice (Bob) and Charlie is $l_{a}\left(l_{b}\right)$. In our scheme, the channel transmittance between Alice (Bob) and Charlie is $\eta_{a}=\eta_{d} \times 10^{-\alpha l_{a} / 10}\left(\eta_{b}=\eta_{d} \times 10^{-\alpha l_{b} / 10}\right)$, where $\eta_{d}$ is the detection efficiency of Charlie's detectors and $\alpha$ is the attenuation coefficient of the ultra-low fiber. In addition, let us suppose that $p_{d}$ is the dark count rate of one detector. For two detectors used by Charlie, we derive the total dark count rate as $2 p_{d}$ and the error rate of background $e_{0}=\frac{1}{2}$.

In Charlie's laboratory, after the BS (see Figure 1), the optical intensities received by detector 1 and detector 2 are given by $D_{1}=\left(\frac{\sqrt{\mu_{a} \eta_{a}}}{2}+\frac{\sqrt{\mu_{b} \eta_{b}}}{2} \cos \theta\right)^{2}$ and $D_{2}=\left(\frac{\sqrt{\mu_{a} \eta_{a}}}{2}-\right.$ $\left.\frac{\sqrt{\mu_{b} \eta_{b}}}{2} \cos \theta\right)^{2}$, where $\theta$ denotes the relative phase between Alice's and Bob's weak coherent states. In our asymmetric protocol, we have $\theta \in\{0, \pi\}$. Thus, the detection probability of each detector is: $Q_{1}=1-\left(1-p_{d}\right) e^{-D_{1}}, Q_{2}=1-\left(1-p_{d}\right) e^{-D_{2}}$.

The gain of the whole system for Charlie's detections can be calculated by $Q_{\mu}=Q_{1}\left(1-Q_{2}\right)+Q_{2}\left(1-Q_{1}\right)+Q_{1} Q_{2}$ and the error rate of the total gain can be derived by $E_{\mu} Q_{\mu}=e_{d} Q_{1}\left(1-Q_{2}\right)+\left(1-e_{d}\right) Q_{2}\left(1-Q_{1}\right)+\frac{1}{2} Q_{1} Q_{2}$, where $e_{d}$ is the misalignment error rate of detectors.

\subsection{Finite-Key Analysis Method for Our Protocol}

Considering the finite-key effects, let $n_{\mu}=N Q_{\mu}$ be the observed number of bits, where $N$ is the number of optical pulses sent by Alice and Bob. By using the random sampling without replacement [47], one can calculate the upper bound of hypothetically observed error rate associated with $E_{\mu}$ with a failure probability $\epsilon_{R S}$ :

$$
\overline{E_{\mu}}=E_{\mu}+\gamma\left(n_{\mu}-k, k, E_{\mu}, \epsilon_{R S}\right),
$$


where $k$ is the number of bits in the chosen time slots at Step 3 (parameter estimation).

In the following, we assume the protocol is $\epsilon$-secure [49] where the maximum failure probability of practical protocol is $\epsilon$. According to universally composable security [50],

$$
\epsilon=\epsilon_{R S}+\bar{\epsilon}+\epsilon_{E C}+\epsilon_{P A}
$$

where $\bar{\epsilon}$ represents the accuracy of estimating the smooth min-entropy. In addition, $\epsilon_{E C}$ corresponds to the probability that error correction fails and $\epsilon_{P A}$ is the probability that privacy amplification fails.

Then we obtain the key rate formula in finite-sized key region, which reads

$$
\begin{aligned}
R_{\mathrm{QSS}}= & Q_{\mu}\left[-\left(1-2 \mu_{\mathrm{max}}\right) \log _{2}\left(P_{\mathrm{co}}\right)-f_{e} h\left(E_{\mu}\right)\right] \\
& -\frac{7}{N} \sqrt{n_{\mu} \log _{2} \frac{2}{\bar{\epsilon}}}-\frac{1}{N} \log _{2} \frac{2}{\epsilon_{E C}}-\frac{2}{N} \log _{2} \frac{1}{\epsilon_{P A}} .
\end{aligned}
$$

Here, $f_{e}$ is the error correction efficiency and $h(x)=-x \log _{2}(x)-(1-x) \log _{2}(1-x)$ is Shannon entropy. $P_{\mathrm{co}}$ is the upper bound of collision probability when considering individual attacks, which can be concluded as $P_{\mathrm{co}}=1-{\overline{E_{\mu}}}^{2}-\left(1-6 \overline{E_{\mu}}\right)^{2} / 2$ [51].

\subsection{Results of Simulation}

We use the genetic algorithm to run the numerical simulations, and the key rate is optimized over the free parameters. Here, we set $\epsilon_{R S}=\bar{\epsilon}=\epsilon_{E C}=\epsilon_{P A}=10^{-10}$ and utilize experimental parameters listed in Table 2. Figure 3 shows how the key rate varies with transmission distance between Alice and Bob when their channels have the same transmittance, where the total pulses are set as $N=10^{12}, N=10^{10}, N=10^{8}$, respectively. We can find that our protocol shows great robustness against finite-key effects. In Figure 4, we plot the results of our asymmetric protocol when the total pulses are set $N=10^{12}$, where the difference in length between Alice's channel and Bob's is fixed at $10 \mathrm{~km}, 50 \mathrm{~km}$, and $100 \mathrm{~km}$, respectively.

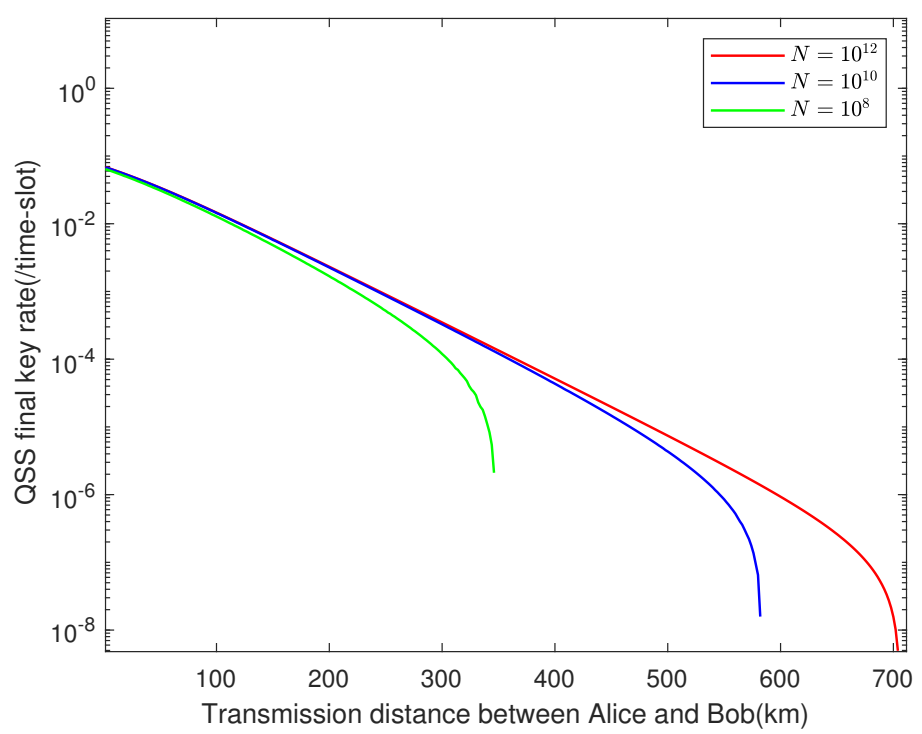

Figure 3. The performance of our protocol under symmetric channels. Under the experimental parameters listed in Table 2, we simulate results in the case that $l_{a}=l_{b}$, where $N=10^{12}, N=10^{10}$, and $N=10^{8}$. 


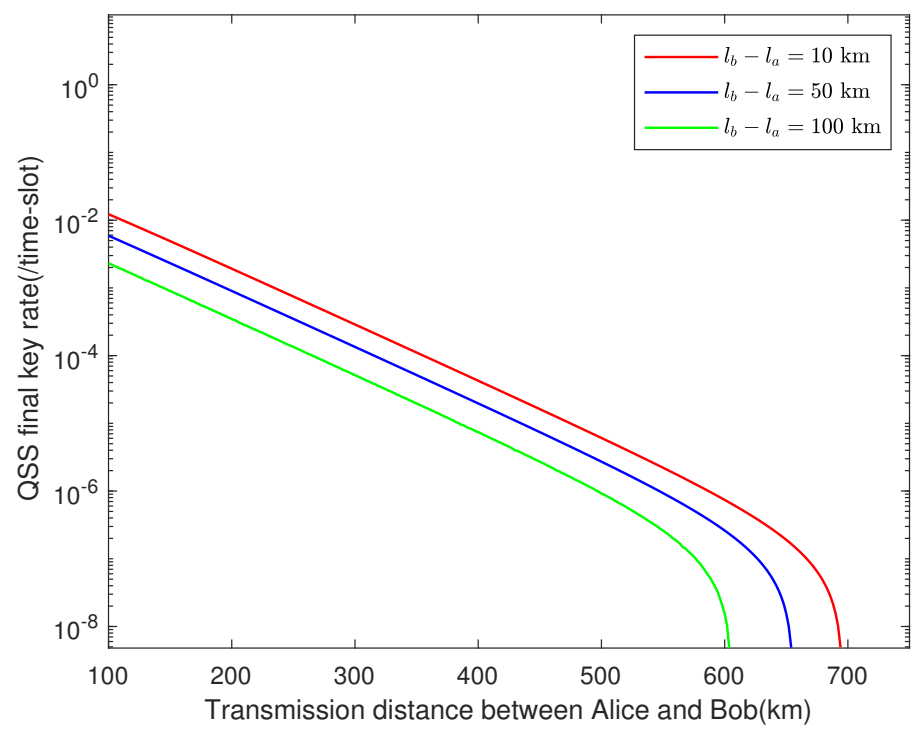

Figure 4. The performance of our protocol under asymmetric channels. Under the experimental parameters listed in Table 2, we simulate results in the case that $N=10^{12}$, where $l_{b}-l_{a}=10,50,100 \mathrm{~km}$.

When the original TF-DPSQSS [29] protocol is applied to the asymmetric channels, a high system error rate will arise since different channel transmittances will lead to the poor performance of interference at the beam splitter. Figure 5 presents numerical results of our asymmetric protocols and the original TF-DPSQSS [29] protocol with the difference in length between two channels fixed at $10 \mathrm{~km}$ and $14 \mathrm{~km}$. We can see clearly from Figure 5 that our asymmetric protocol improves the secret key rate by two orders of magnitude when $l_{b}-l_{a}=14 \mathrm{~km}$. Moreover, no keys are obtained with difference fixed at 50,100 km in the original protocol, whereas Figure 4 shows that our protocol still provides a high key rate when the difference is large. It means that in the asymmetric channels, the performance of the asymmetric protocol is much better than that of the original protocol, especially when channels are extremely asymmetric.

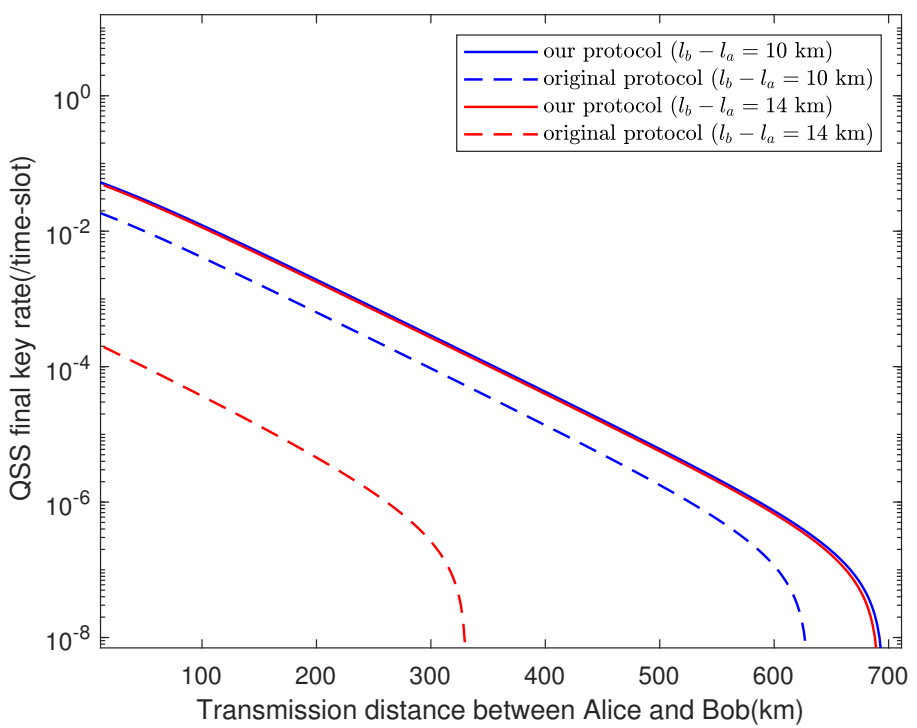

Figure 5. Quantum secret sharing key rate vs transmission distance between Alice and Bob. Under the experimental parameters listed in Table 2, we compare the simulation results of the original TF-DPSQSS [29] protocol and our asymmetric protocol with $N=10^{12}$, where the difference in length between Alice's and Bob's channels is fixed at $10 \mathrm{~km}, 14 \mathrm{~km}$. 
Table 2. Simulation parameters. $\eta_{d}$ and $p_{d}$ are the detection efficiency and dark count rate. $\alpha$ is the attenuation coefficient of the ultra-low fiber. $f_{e}$ is the error correction efficiency.

\begin{tabular}{cccc}
\hline$\eta_{d}$ & $p_{d}$ & $\alpha$ & $f_{e}$ \\
\hline $55 \%$ & $10^{-8}$ & 0.165 & 1.15 \\
\hline
\end{tabular}

\section{Conclusions}

In summary, we propose a differential phase shift quantum secret sharing protocol over asymmetric channels and give the security proof of our protocol against individual attacks. Moreover, we extend the asymptotic key rate of TF-DPSQSS [29] to finite-key region. Through implementing free parameter optimization on the numerical simulations, we demonstrate that our asymmetric protocol can dramatically improve the key generation rate and the transmission distance compared with the original TF-DPSQSS [29] protocol. As we have shown before, when the difference in length between Alice's channel and Bob's is fixed at $14 \mathrm{~km}$, the key rate of the asymmetric protocol is two orders of magnitude higher than the original TF-DPSQSS [29] protocol. Furthermore, our protocol obtains a high key rate when the difference is large. In addition, it is convenient and efficient to implement by allowing Alice and Bob to set asymmetric intensities independently, especially in a network setting. Due to the remarkable performance of our asymmetric protocol, it can be applied directly to the QSS experiments over asymmetric channels and represents a further step towards practical application of QSS.

Author Contributions: Z.-Y.J. and J.G. contributed equally to this work. All the authors contributed to the refinement and generalization of the concept and its presentation of the present paper. H.-L.Y. and Z.-B.C. supervised the whole project. All authors have read and agreed to the published version of the manuscript.

Funding: National Natural Science Foundation of China (61801420); Key-Area Research and Development Program of Guangdong Province (2020B0303040001); Fundamental Research Funds for the Central Universities (020414380182, 020414380141).

Institutional Review Board Statement: Not applicable.

Informed Consent Statement: Not applicable.

Data Availability Statement: Data underlying the results presented in this paper are not publicly available at this time, but may be obtained from the authors upon reasonable request.

Conflicts of Interest: The authors declare no conflict of interest.

\section{References}

1. Shamir, A. How to share a secret. Commun. ACM 1979, 22, 612-613. [CrossRef]

2. Blakley, G.R. Safeguarding cryptographic keys. In Proceedings of the 1979 AFIPS National Computer Conference, New York, NY, USA, 4-7 June 1979; Volume 48, pp. 313-317.

3. Brickell, E.F. Some ideal secret sharing schemes. In Workshop on the Theory and Application of of Cryptographic Techniques; Springer: Berlin/Heidelberg, Germany, 1989; pp. 468-475.

4. Shor, P. Algorithms for quantum computation: Discrete logarithms and factoring. In Proceedings of the 35th Annual Symposium on Foundations of Computer Science, Santa Fe, NM, USA, 20-22 November 1994; pp. 124-134.

5. Hillery, M.; Bužek, V.; Berthiaume, A. Quantum secret sharing. Phys. Rev. A 1999, 59, 1829. [CrossRef]

6. Xiao, L.; Long, G.L.; Deng, F.G.; Pan, J.W. Efficient multiparty quantum-secret-sharing schemes. Phys. Rev. A 2004, 69, 052307. [CrossRef]

7. Yu, I.C.; Lin, F.L.; Huang, C.Y. Quantum secret sharing with multilevel mutually (un) biased bases. Phys. Rev. A 2008, 78, 012344. [CrossRef]

8. Nascimento, A.C.A.; Mueller-Quade, J.; Imai, H. Improving quantum secret-sharing schemes. Phys. Rev. A 2001, 64, 042311. [CrossRef]

9. Singh, S.K.; Srikanth, R. Generalized quantum secret sharing. Phys. Rev. A 2005, 71, 012328. [CrossRef]

10. Keet, A.; Fortescue, B.; Markham, D.; Sanders, B.C. Quantum secret sharing with qudit graph states. Phys. Rev. A 2010, 82, 062315. [CrossRef] 
11. Fu, Y.; Yin, H.L.; Chen, T.Y.; Chen, Z.B. Long-distance measurement-device-independent multiparty quantum communication. Phys. Rev. Lett. 2015, 114, 090501. [CrossRef] [PubMed]

12. Rahaman, R.; Parker, M.G. Quantum scheme for secret sharing based on local distinguishability. Phys. Rev. A 2015, 91, 022330. [CrossRef]

13. Tavakoli, A.; Herbauts, I.; Żukowski, M.; Bourennane, M. Secret sharing with a single $d$-level quantum system. Phys. Rev. A 2015, 92, 030302. [CrossRef]

14. Kogias, I.; Xiang, Y.; He, Q.; Adesso, G. Unconditional security of entanglement-based continuous-variable quantum secret sharing. Phys. Rev. A 2017, 95, 012315. [CrossRef]

15. Qin, H.; Tang, W.K.S.; Tso, R. Hierarchical Quantum Secret Sharing Based On Special High-Dimensional Entangled State. IEEE J. Sel. Top. Quantum Electron. 2020, 26, 6600106. [CrossRef]

16. Tittel, W.; Zbinden, H.; Gisin, N. Experimental demonstration of quantum secret sharing. Phys. Rev. A 2001, 63, 042301. [CrossRef]

17. Chen, Y.A.; Zhang, A.N.; Zhao, Z.; Zhou, X.Q.; Lu, C.Y.; Peng, C.Z.; Yang, T.; Pan, J.W. Experimental quantum secret sharing and third-man quantum cryptography. Phys. Rev. Lett. 2005, 95, 200502. [CrossRef] [PubMed]

18. Gaertner, S.; Kurtsiefer, C.; Bourennane, M.; Weinfurter, H. Experimental Demonstration of Four-Party Quantum Secret Sharing. Phys. Rev. Lett. 2007, 98, 020503. [CrossRef]

19. Bell, B.; Markham, D.; Herrera-Martí, D.; Marin, A.; Wadsworth, W.; Rarity, J.; Tame, M. Experimental demonstration of graph-state quantum secret sharing. Nat. Commun. 2014, 5, 5480. [CrossRef]

20. Inoue, K.; Ohashi, T.; Kukita, T.; Watanabe, K.; Hayashi, S.; Honjo, T.; Takesue, H. Differential-phase-shift quantum secret sharing. Opt. Express 2008, 16, 15469-15476. [CrossRef]

21. Inoue, K.; Waks, E.; Yamamoto, Y. Differential Phase Shift Quantum Key Distribution. Phys. Rev. Lett. 2002, 89, 037902. [CrossRef]

22. Inoue, K.; Waks, E.; Yamamoto, Y. Differential-phase-shift quantum key distribution using coherent light. Phys. Rev. A 2003, 68, 022317. [CrossRef]

23. Honjo, T.; Inoue, K.; Takahashi, H. Differential-phase-shift quantum key distribution experiment with a planar light-wave circuit Mach-Zehnder interferometer. Opt. Lett. 2004, 29, 2797-2799. [CrossRef]

24. Takesue, H.; Diamanti, E.; Honjo, T.; Langrock, C.; Fejer, M.M.; Inoue, K.; Yamamoto, Y. Differential phase shift quantum key distribution experiment over $105 \mathrm{~km}$ fibre. New J. Phys. 2005, 7, 232. [CrossRef]

25. Waks, E.; Takesue, H.; Yamamoto, Y. Security of differential-phase-shift quantum key distribution against individual attacks. Phys. Rev. A 2006, 73, 012344. [CrossRef]

26. Diamanti, E.; Takesue, H.; Langrock, C.; Fejer, M.M.; Yamamoto, Y. $100 \mathrm{~km}$ differential phase shift quantum key distribution experiment with low jitter up-conversion detectors. Opt. Express 2006, 14, 13073-13082. [CrossRef] [PubMed]

27. Takeoka, M.; Guha, S.; Wilde, M.M. Fundamental rate-loss tradeoff for optical quantum key distribution. Nat. Commun. 2014, 5, 5235. [CrossRef]

28. Pirandola, S.; Laurenza, R.; Ottaviani, C.; Banchi, L. Fundamental limits of repeaterless quantum communications. Nat. Commun. 2017, 8, 15043. [CrossRef] [PubMed]

29. Gu, J.; Cao, X.Y.; Yin, H.L.; Chen, Z.B. Differential phase shift quantum secret sharing using a twin field. Opt. Express 2021, 29, 9165-9173. [CrossRef] [PubMed]

30. Lucamarini, M.; Yuan, Z.L.; Dynes, J.F.; Shields, A.J. Overcoming the rate-distance limit of quantum key distribution without quantum repeaters. Nature 2018, 557, 400-403. [CrossRef]

31. Ma, X.; Zeng, P.; Zhou, H. Phase-matching quantum key distribution. Phys. Rev. X 2018, 8, 031043. [CrossRef]

32. Wang, X.B.; Yu, Z.W.; Hu, X.L. Twin-field quantum key distribution with large misalignment error. Phys. Rev. A 2018, $98,062323$. [CrossRef]

33. Lin, J.; Lütkenhaus, N. Simple security analysis of phase-matching measurement-device-independent quantum key distribution. Phys. Rev. A 2018, 98, 042332. [CrossRef]

34. Yin, H.L.; Fu, Y. Measurement-device-independent twin-field quantum key distribution. Sci. Rep. 2019, 9, 3045. [CrossRef] [PubMed]

35. Cui, C.; Yin, Z.Q.; Wang, R.; Chen, W.; Wang, S.; Guo, G.C.; Han, Z.F. Twin-Field Quantum Key Distribution without Phase Postselection. Phys. Rev. Appl. 2019, 11, 034053. [CrossRef]

36. Curty, M.; Azuma, K.; Lo, H.K. Simple security proof of twin-field type quantum key distribution protocol. NPJ Quantum Inf. 2019, 5, 64. [CrossRef]

37. Yin, H.L.; Chen, Z.B. Coherent-state-based twin-field quantum key distribution. Sci. Rep. 2019, 9, 14918. [CrossRef] [PubMed]

38. Hu, X.L.; Jiang, C.; Yu, Z.W.; Wang, X.B. Sending-or-not-sending twin-field protocol for quantum key distribution with asymmetric source parameters. Phys. Rev. A 2019, 100, 062337. [CrossRef]

39. Grasselli, F.; Navarrete, Á.; Curty, M. Asymmetric twin-field quantum key distribution. New J. Phys. 2019, 21, 113032. [CrossRef]

40. Maeda, K.; Sasaki, T.; Koashi, M. Repeaterless quantum key distribution with efficient finite-key analysis overcoming the rate-distance limit. Nat. Commun. 2019, 10, 3140. [CrossRef] [PubMed]

41. Minder, M.; Pittaluga, M.; Roberts, G.; Lucamarini, M.; Dynes, J.; Yuan, Z.; Shields, A. Experimental quantum key distribution beyond the repeaterless secret key capacity. Nat. Photonics 2019, 13, 334-338. [CrossRef]

42. Liu, Y.; Yu, Z.W.; Zhang, W.; Guan, J.Y.; Chen, J.P.; Zhang, C.; Hu, X.L.; Li, H.; Jiang, C.; Lin, J.; et al. Experimental Twin-Field Quantum Key Distribution through Sending or Not Sending. Phys. Rev. Lett. 2019, 123, 100505. [CrossRef] 
43. Zhong, X.; Hu, J.; Curty, M.; Qian, L.; Lo, H.K. Proof-of-Principle Experimental Demonstration of Twin-Field Type Quantum Key Distribution. Phys. Rev. Lett. 2019, 123, 100506. [CrossRef]

44. Zhong, X.; Wang, W.; Qian, L.; Lo, H.K. Proof-of-principle experimental demonstration of twin-field quantum key distribution over optical channels with asymmetric losses. NPJ Quantum Inf. 2021, 7, 8. [CrossRef]

45. Tomamichel, M.; Lim, C.C.W.; Gisin, N.; Renner, R. Tight finite-key analysis for quantum cryptography. Nat. Commun. 2012, 3, 634. [CrossRef] [PubMed]

46. Grasselli, F.; Kampermann, H.; Bruß, D. Finite-key effects in multipartite quantum key distribution protocols. New J. Phys. 2018, 20, 113014. [CrossRef]

47. Yin, H.L.; Zhou, M.G.; Gu, J.; Xie, Y.M.; Lu, Y.S.; Chen, Z.B. Tight security bounds for decoy-state quantum key distribution. Sci. Rep. 2020, 10, 14312. [CrossRef]

48. Yin, H.L.; Chen, Z.B. Finite-key analysis for twin-field quantum key distribution with composable security. Sci. Rep. 2019, 9, 17113. [CrossRef]

49. Müller-Quade, J.; Renner, R. Composability in quantum cryptography. New J. Phys. 2009, 11, 085006. [CrossRef]

50. Canetti, R. Universally composable security: A new paradigm for cryptographic protocols. In Proceedings of the 42nd IEEE Symposium on Foundations of Computer Science, Newport Beach, CA, USA, 8-11 October 2001; pp. $136-145$.

51. Lütkenhaus, N. Estimates for practical quantum cryptography. Phys. Rev. A 1999, 59, 3301. [CrossRef] 